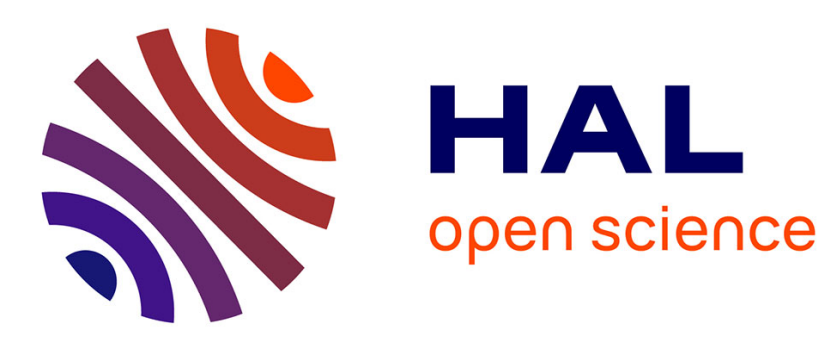

\title{
Nonlinear state estimation using an invariant unscented Kalman filter
}

\author{
Jean-Philippe Condomines, Cédric Seren, Gautier Hattenberger
}

\section{To cite this version:}

Jean-Philippe Condomines, Cédric Seren, Gautier Hattenberger. Nonlinear state estimation using an invariant unscented Kalman filter. AIAA GNC 2013, AIAA Guidance, Navigation and Control Conference, Aug 2013, Boston, United States. pp 1-15; ISBN : 978-1-62410-224-0, 10.2514/6.20134869. hal-00933568

\section{HAL Id: hal-00933568 \\ https://hal-enac.archives-ouvertes.fr/hal-00933568}

Submitted on 3 Feb 2014

HAL is a multi-disciplinary open access archive for the deposit and dissemination of scientific research documents, whether they are published or not. The documents may come from teaching and research institutions in France or abroad, or from public or private research centers.
L'archive ouverte pluridisciplinaire HAL, est destinée au dépôt et à la diffusion de documents scientifiques de niveau recherche, publiés ou non, émanant des établissements d'enseignement et de recherche français ou étrangers, des laboratoires publics ou privés. 


\title{
Nonlinear state estimation using an invariant unscented Kalman filter
}

\author{
Jean-Philippe Condomines * \\ Ecole Nationale de l'Aviation Civile - BP 54005, Toulouse Cedex 4, 31055, France \\ Cédric Seren † \\ ONERA - The French Aerospace Lab - BP 74025, Toulouse Cedex 4, 31055, France \\ Gautier Hattenberger $\ddagger$ \\ Ecole Nationale de l'Aviation Civile - BP 54005, Toulouse Cedex 4, 31055, France
}

In this paper, we proposed a novel approach for nonlinear state estimation, named $\pi$-IUKF (Invariant Unscented Kalman Filter), which is based on both invariant filter estimation and UKF theoretical principles. Several research works on nonlinear invariant observers have been led and provide a geometrical-based constructive method for designing filters dedicated to nonlinear state estimation problems while preserving the physical properties and systems symmetries. The general invariant observer guarantees a straightforward form of the nonlinear estimation error dynamics whose properties are remarkable. The developed $\pi$-IUKF estimator suggests a systematic approach to determine all the symmetry-preserving correction terms, associated with a nonlinear state-space representation used for prediction, without requiring any linearization of the differential equations. The exploitation of the UKF principles within the invariant framework has required the definition of a compatibility condition on the observation equations. As a first result, the estimated covariance matrices of the $\pi$-IUKF converge to constant values due to the symmetry-preserving property provided by the nonlinear invariant estimation theory. The designed $\pi$-IUKF method has been successfully applied to some relevant practical problems such as the estimation of Attitude and Heading for aerial vehicles using low-cost $\mathrm{AH}$ reference systems (i.e., inertial/magnetic sensors characterized by low performances).

\section{Nomenclature}

$e_{i} \quad$ Orthonormal basis vector, $i \in \llbracket 1 ; 3 \rrbracket$

$\omega$ Instantaneous angular velocity vector, $\mathrm{rad} / \mathrm{s}$

$\omega_{0} \quad$ Reference angular velocity vector in $\mathbb{R}^{3}, \mathrm{rad} / \mathrm{s}$

$a_{0}, b_{0}$ Positive scalars

$q_{0} \quad$ Unit quaternion

$\phi \quad$ Bank angle

$\theta \quad$ Pitch angle

$\psi \quad$ Heading angle

$u \quad$ Longitudinal speed component (body frame)

$v \quad$ Lateral speed component (body frame)

$w \quad$ Vertical speed component (body frame)

$A \quad$ Constant gravity vector in the North-East-Down (NED) coordinate system i.e., $A=g e_{3}, m / s^{2}$

a Specific acceleration vector, $\mathrm{m} / \mathrm{s}^{2}$

$V \quad$ Velocity vector of the center of gravity (Earth frame), $\mathrm{m} / \mathrm{s}$

*PhD student, Ecole Nationale de l'Aviation Civile (ENAC), jean-philippe.condomines@recherche.enac.fr.

$\dagger$ Doctor and research engineer, ONERA - Control Systems and Flight Dynamics Department, cedric.seren@onera.fr.

$\ddagger$ Doctor, Ecole Nationale de l’Aviation Civile (ENAC), gautier.hattenberger@recherche.enac.fr. 


\section{Introduction}

The attitude estimation problem for nonlinear dynamic system is an important research topic and is a major concern in the aerospace engineering community. ${ }^{1}$ The Extended Kalman Filter (EKF), and its numerous variants e.g., the multiplicative EKF, is the most widely used signal processing methodology. Its principle relies on a first-order linearization of the nonlinear model equations so that the traditional linear Kalman Filter (KF) can be applied. However, it has two well known drawbacks: ${ }^{2}$ (1) the first-order linearization can introduce large errors in the mean and covariance of the state vector and (2) the calculation of Jacobian matrices is nontrivial for many applications. To overcome this problem, the Unscented $\mathrm{KF}^{3}$ (UKF) is an efficient derivative free estimation algorithm for determining approximate solutions to discretetime nonlinear optimal filtering problems. It has been successfully applied to various practical problems and it has been shown that it outperforms the EKF in many cases. ${ }^{4-6}$

Recently, several research works on nonlinear invariant observers have been led and provide a geometricalbased constructive method for designing filters dedicated to the state estimation problem of dynamical systems while preserving the physical properties and systems symmetries. Such an approach can be very tedious and non-systematic for complex dynamical systems. That's why, a more general EKF-based method dedicated to gains calculation (and which exploits a re-linearization of the invariant state estimation error dynamics) has been recently developed in. ${ }^{7-9}$ Unfortunately, the Invariant EKF (IEKF) is based on a kind of second-order approximations of the invariant estimation error. Once again, this technique can be very complex to implement due to this re-linearization.

The contribution of this paper is to propose a novel and original approach for nonlinear state estimation, named $\pi$-IUKF (Invariant Unscented Kalman Filter), which is based on both invariant filter estimation and UKF theoretical principles. The developed $\pi$-IUKF estimator suggests a systematic approach to determine all the symmetry-preserving correction terms, associated with a given nonlinear state-space representation used for state prediction, without requiring any linearization of the dynamical systems equations. It is named " $\pi$-IUKF" because a compatibility condition, which makes use of a specific nonlinear function denoted " $\pi$ ", on the observation equations has been defined and integrated to the standard UKF algorithm.

The paper is organized as follows: in section II, kinematics and nonlinear evolution equations are presented and the $\pi$-Invariant Unscented Kalman Filter is detailed in section III. To conclude, section IV shows some results obtained in simulation and validate the whole approach.

\section{Dynamical system modeling}

In order to tackle a wide range of applications, various implementations of flight dynamics models, in terms of assumptions and numerical techniques, therefore exist. Mini-Unmanned Aerial Vehicles (miniUAVs) are commonly represented using a 6 Degrees of Freedom (DoF) quaternion-based kinematic model (3 DoF correspond to the translational motion $(u, v, w)$ and the 3 remaining DoF to the rotational motion $(\phi, \theta, \psi))$. This choice is due to:

1. the high dynamical behavior of mini-UAVs which causes problems when using the typical Euler angles representation;

2. the difficulty for such systems to derive a reliable and representative aerodynamic modeling from scratch (see Fig.1) (which could be included within a full nonlinear flight dynamics model) since mini-UAVs must remain low-cost experimental means (no CFD calculations, no wind-tunnel tests, ... ).
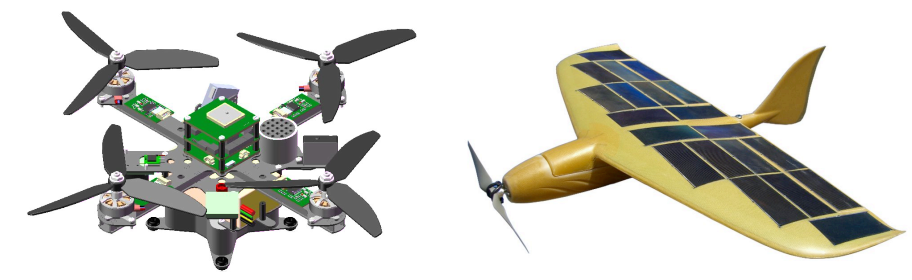

Figure 1. Quadrirotor (Blender, $350 \mathrm{~mm}$ diameter and $300 \mathrm{~g}$ weight) and aircraft (Solar Storm, $500 \mathrm{~mm}$ span and $400 \mathrm{~g}$ weight) min-UAVs from the ENAC (Ecole Nationale de l'Aviation Civile) University 


\section{A. Kinematics and Nonlinear Evolution Equations}

Assuming a flat non-rotating Earth, the flying rigid body motion can be mathematically described by:

$$
\left\{\begin{array}{l}
\dot{q}=\frac{1}{2} q * \omega \\
\dot{V}=A+q * a * q^{-1}
\end{array}\right.
$$

In Eq.(1), symbol $*$ corresponds to the quaternion product. The first expression of Eq.(1) describes the kinematic relation of the body of the considered mini-UAV and the second one corresponds to the Newton's law. ${ }^{10}$ The instantaneous attitude of the flying mini-UAV can be generalized and deduced using the aforementioned standard quaternionial form which provides at the same time:

- a global parametrization;

- avoids the mathematical singularities inherent to Euler angles;

- and is convenient for calculations and simulations.

For more details about formulas used on quaternion in this paper, see Appendix I.

\section{B. Comments on the Measurement and Sensor Reliability}

Recent progresses in the miniaturization of the inertial instruments i.e., Inertial Navigation System (INS) and Global Positioning System (GPS) hardware have led to the design of small, low-cost integrated navigation systems. Under conditions where GPS is available, this one provides a position accuracy of approximately 3 to 10 meters. In our case, where the position is unavailable or intermittent such as in urban or indoor environments, a major issue for autonomous maneuvering and obstacles avoidance for mini-UAVs is to estimate vehicle orientation with respect to an inertial reference frame. Thus navigation quality is limited by inertial sensor performance: given the size, power and cost constraints of miniature systems. To recover navigation accuracy using low-cost sensors such as the well-known non-aided Attitude and Heading Reference Systems (AHRS), it is necessary to use, if possible, additional instruments (e.g., magnetometers, which are used to increase the heading accuracy) and/or nonlinear estimation algorithm to improve the global miniUAVs handling qualities. The nonlinear state estimation makes use of 3 triaxial sensors which deliver 9 scalar measurement signals:

- 3 of them are associated with 3 magnetometers which provide a local measurement of Earth's magnetic field, which is known constant ${ }^{a}$ and expressed in the body-fixed frame such that the vector $y_{B}=$ $q^{-1} * B * q$ where $B=\left[B_{x}, B_{y}, B_{z}\right]$ can be considered as an output of the observation equations;

- 3 gyroscopes allow to obtain a measurement of the instantaneous angular velocity vector denoted by $\omega_{m} \in \mathbb{R}^{3}$ s.t $\omega_{m}=\left[\omega_{m x}, \omega_{m y}, \omega_{m z}\right]^{T}$

- and finally, 3 accelerometers give a measurement of the specific acceleration denoted by $a_{m} \in \mathbb{R}^{3}$ s.t $a_{m}=\left[a_{m x}, a_{m y}, a_{m z}\right]^{T}$.

All these measurements are obviously corrupted by noises whose effects will be taken into account in §III-B. In this non-aided AHRS, no velocity and position measurements are available (no GPS, no Pitot sensors). Thus, to keep the whole nonlinear state representation observable given these available informations, the assumption that the linear acceleration $\dot{V}$ remains small is made, i.e., $\dot{V}=0$. As a result, the specific acceleration vector, expressed in the body-fixed frame, can be approximated by $a=-y_{A}=-q^{-1} * A * q$ where $A$ is the local Earth's gravity vector. This one can be compared, as an another output vector, with its corresponding measurement $a_{m}$. Thereby, we consider the following system $\mathcal{M}_{s}$ given by:

$$
\mathcal{M}_{s}\left\{\begin{array}{l}
\dot{q}=\frac{1}{2} q * \omega \\
y=\left(\begin{array}{l}
y_{A} \\
y_{B}
\end{array}\right)=\left(\begin{array}{l}
q^{-1} * A * q \\
q^{-1} * B * q
\end{array}\right)
\end{array}\right.
$$

${ }^{a}$ The magnetic field can be determined from a world magnetic model such as B is equal to [0.5156 0.0570 0.8549] at the local flight coordinates $43.617-43^{\circ}-37^{\prime}(\mathrm{N})$ and $1.450-1^{\circ}-27^{\prime}(\mathrm{E})$ 
All the sensors embedded are low-cost and so have imperfections. The major error sources in the navigation system are due to: - the inertial instrument imperfection; - the possible incorrect navigation system initialization (e.g., on magnetometers); - and the imperfections in the local Earth's gravity vector used in computations. For both instruments, the largest errors are usually a bias instability (expressed respectively in (deg/hr) for gyros and $\mu \mathrm{g}$ for the accelerometers biases) and a wrong scale-factor value. Obviously, smaller will be the inertial sensor errors and better will be the quality of the instruments (improved accuracy of the navigation results), but higher will be the cost of the system. Fig. 2 from ${ }^{11}$ shows INS relative strapdown system cost "projections" as a function of the inertial instruments technology and performance. Note that colored areas are function of the quantity of sensor systems produced which influences their production cost. Thus, only MEMS (Micro-Electro-Mechanical Systems) gyros and accelerometers (performing at around $100^{\circ} / \mathrm{hr}$ and $100 \mathrm{mg}$ bias stabilities respectively) are currently suitable for a potential use as a low-cost AHRS.

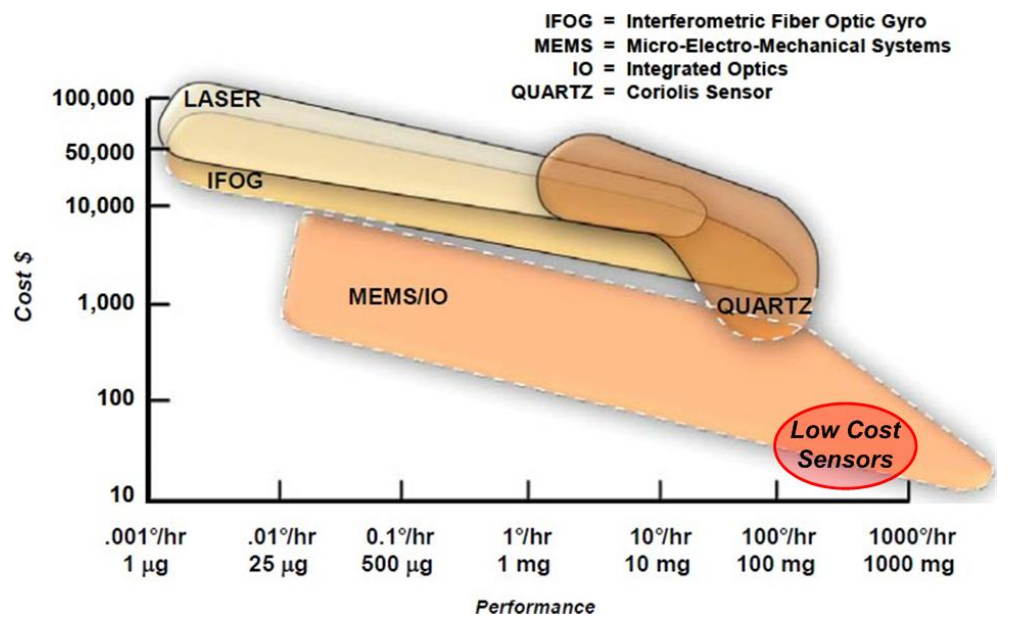

Figure 2. Strapdown INS cost as a function of instrument technology

Based on these technical elements and the observation relations which composed $\mathcal{M}_{s}$, it is possible to choose how these imperfections can be modeled since some degrees of freedom exist. A first-order observability analysis - which can be led analytically by linearizing $\mathcal{M}_{s}-\left(\operatorname{see}^{12}\right.$ for more details) shows that 6 additional unknown constants can be estimated without introducing inobservability. Thus, an additive constant bias vector $\omega_{b}$ is basically considered on the angular velocity vector measurement $\omega_{m}$. Then, for mini-UAV safety (magnetic measurements used to estimate the mini-UAV's heading only and accelerometric imperfection estimated without relying on the disturbed magnetic measurement), only two more constant positive scaling factors $a_{s}$ and $b_{s}$ are introduced in the estimation scheme. The latter quantity $b_{s}$ will be used to adjust and preserve the unit norm on vector $y_{B}$. All these sensor imperfections are modeled as gaussian random walks which can be physically interpreted as slowly varying parameters. The $\mathcal{M}_{s}$ modeling now becomes:

$$
\mathcal{M}_{s}^{+}\left\{\begin{array}{l}
\dot{q}=\frac{1}{2} q *\left(\omega_{m}-\omega_{b}\right) \\
\dot{\omega}_{b}=0 \\
\dot{a}_{s}=0 \\
\dot{b}_{s}=0 \\
\left(\begin{array}{c}
y_{A} \\
y_{B}
\end{array}\right)=\left(\begin{array}{l}
a_{s} q^{-1} * A * q \\
b_{s} q^{-1} * B * q
\end{array}\right) \quad \text { (ebolution) }
\end{array}\right.
$$

where $\omega_{m}$ is seen as an imperfect and noisy but known measured input and $a_{m}$ as an available imperfect and noisy measured output like $B$. The nonlinear state space representation corresponding to $\mathcal{M}_{s}^{+}$can be 
described in a compact form such as: $\dot{x}=f(x, u)$ and $y=h(x, u)$ where: $x=\left[q^{T}, \omega_{b}^{T}, a_{s}, b_{s}\right]^{T}, u=\left[\omega_{m}\right]$ and $y=\left[y_{A}^{T}, y_{C}^{T}\right]$ are the state, input and output vectors respectively.

\section{III. $\pi$-Invariant Unscented Kalman Filter}

\section{A. Invariant observer and system symmetries}

This section presents briefly the main theoretical principles of some research works dealing with dynamical system symmetries and invariant observer. ${ }^{13}$ Let's consider the following nonlinear state-space representation defined by:

$$
\left\{\begin{array}{l}
\frac{d x}{d t}=f(x, u) \\
y=h(x, u)
\end{array}\right.
$$

where the state (resp. input)(resp. output) vector belongs to an open set $\mathcal{X} \in \mathbb{R}^{n}$ (resp. $\mathcal{U} \in \mathbb{R}^{m}$ ) (resp. $\left.\mathcal{Y} \in \mathbb{R}^{p}, p \leq n\right)$. Then, we consider the transformation group defined on $\mathcal{X} \times \mathcal{U} \times \mathcal{Y}$ such that :

$$
\begin{aligned}
G \times(\mathcal{X} \times \mathcal{U} \times \mathcal{Y}) & \rightarrow(\mathcal{X} \times \mathcal{U} \times \mathcal{Y}) \\
(g, x, u, y) & \mapsto\left(\varphi_{g}(x), \psi_{g}(u), \rho_{g}(y)\right)=(X, U, Y)
\end{aligned}
$$

where $G$ is a Lie-group ${ }^{b}$ (i.e., a finite-dimensional differentiable manifold in which multiplication and inversion group operations are also differentiable) and $\left(\varphi_{g}, \psi_{g}, \rho_{g}\right)$ are three diffeomorphisms ${ }^{\mathrm{c}}$ parametrized by $g \in G$. Practically, the objective is to define 3 specific coordinates transformations whose actions on state, input and output variables respectively are distinct such that the evolution $(d x / d t=f(x, u))$ and observation $(y=h(x, u))$ equations are left unchanged i.e., $\dot{X}=f(X, U)$ and $Y=h(X, U)$.

Thus, a dynamical system $\dot{x}=f(x, u)$ will be said $G$-invariant if $\exists\left(\varphi_{g}, \psi_{g}\right)_{g \in G}, \forall(g, x, u) \in G \times(\mathcal{X} \times \mathcal{U})$, $f\left(\varphi_{g}(x), \psi_{g}(u)\right)=D \varphi_{g}(x) \cdot f(x, u)$ and $G$-equivariant if moreover $\exists\left(\rho_{g}\right)_{g \in G}$ acting on $\mathcal{Y}, \forall(g, x, u) \in G \times$ $(\mathcal{X} \times \mathcal{U}), h\left(\varphi_{g}(x), \psi_{g}(u)\right)=\rho_{g}(h(x, u))$. These last definitions mean that all state and output equations remain explicitly identical. Based on such $G$-invariant/equivariant dynamical systems it is possible to design invariant observers (whose properties are often remarkable) by copying the predetermined group actions on the estimated state and output spaces. By doing this, it is possible to replace the usual linear output error on which a lot of observers are based (for instance KF), and which does not preserve systems symmetries, with a nonlinear invariant one. In our inertial navigation application, this invariance feature corresponds to some physical aspects of the mini-UAV's movement such as the independent choice of the reference frame (Galilean invariances through transformations composed with rotations and translations). So, we are looking for frame transformations that leave the system identical. Considering the explicit $\mathcal{M}_{s}^{+}$modeling given in Eq.(2) and the Lie-group $G=\mathbb{H}_{1} \times \mathbb{R}^{5}$ (where $\mathbb{H}_{1}$ is the unit quaternions manifold) acting on the whole state space of $\mathcal{M}_{s}^{+}$, the following variable transformations give to our considered dynamical system the $G$-invariant

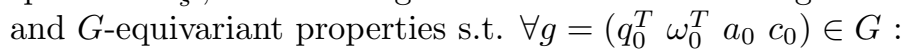

$$
\begin{aligned}
& \text { 1) } \varphi_{g}\left(x=\left(\begin{array}{llll}
q^{T} & \omega_{b}^{T} & a_{s} & b_{s}
\end{array}\right)^{T}\right)=\left(\begin{array}{c}
q * q_{0} \\
q_{0}^{-1} * \omega_{b} * q_{0}+\omega_{0} \\
a_{s} \cdot a_{0} \\
b_{s} \cdot b_{0}
\end{array}\right) \\
& \text { 2) } \psi_{g}\left(\omega=\left(\omega_{m}\right)\right)=q_{0}^{-1} * \omega_{m} * q_{0}+\omega_{0} \\
& \text { 3) } \rho_{g}\left(y=\left(\begin{array}{ll}
y_{A}^{T} & y_{B}^{T}
\end{array}\right)^{T}\right)=\left(\begin{array}{l}
a_{0} \cdot q_{0}^{-1} * y_{A} * q_{0} \\
b_{0} \cdot q_{0}^{-1} * y_{B} * q_{0}
\end{array}\right)
\end{aligned}
$$

These latter are equivalent to time-constant rotations and translations in both earth- and body-fixed frames. Moreover, let's consider $Q=\left(q * q_{0}\right), \Omega_{b}=\left(q_{0}^{-1} * \omega_{b} * q_{0}+\omega_{0}\right)$ and $\Omega_{m}=\left(q_{0}^{-1} * \omega_{m} * q_{0}+\omega_{0}\right)$. The system

\footnotetext{
be.g., $S E_{3}(\mathbb{R})$ (rotation matrices and translation vectors of dimension 3) is a Lie-group.

${ }^{\mathrm{c}}$ Given a Lie-group, it is called diffeomorphism if it is bijective and its inverse is differentiable.
} 
$\mathcal{M}_{s}^{+}$is indeed invariant by transformation group since:

$$
\begin{aligned}
\dot{Q} & =\overbrace{\left(q * q_{0}\right)}=\left(\frac{1}{2} q *\left(\omega_{m}-\omega_{b}\right)\right) * q_{0}=\frac{1}{2}\left(q *\left(\omega_{m}-\omega_{b}\right)\right) * q_{0} \\
& =\frac{1}{2}\left(q * \omega_{m}-q * \omega_{b}\right) * q_{0}=\frac{1}{2}\left(q * \omega_{m} * q_{0}-q * \omega_{b} * q_{0}\right) \\
& =\frac{1}{2}\left(q * q_{0} * q_{0}^{-1} * \omega_{m} * q_{0}-q * q_{0} * q_{0}^{-1} * \omega_{b} * q_{0}\right) \\
& =\frac{1}{2}\left(q * q_{0}\right) *\left(\left(q_{0}^{-1} * \omega_{m} * q_{0}+\omega_{0}\right)-\left(q_{0}^{-1} * \omega_{b} * q_{0}+\omega_{0}\right)\right) \\
& =\frac{1}{2} Q *\left(\left(q_{0}^{-1} * \omega_{m} * q_{0}+\omega_{0}\right)-\left(q_{0}^{-1} * \omega_{b} * q_{0}+\omega_{0}\right)\right)=\frac{1}{2} Q *\left(\Omega_{m}-\Omega_{b}\right) \\
\overbrace{\left(a_{s} \cdot a_{0}\right)}^{\cdot} & =\overbrace{\dot{a}_{s} \cdot a_{0}=0}^{\dot{\Omega}_{b}}=\overbrace{\left(q_{0}^{-1} * \omega_{b} * q_{0}+\omega\right)}=q_{0}^{-1} * \dot{\omega_{b}} * q_{0}=0 \\
\overbrace{\left.b_{s} \cdot b_{0}\right)} & =\dot{b_{s}} \cdot b_{0}=0
\end{aligned}
$$

whereas the output (observation) is G-compatible since:

$$
\begin{aligned}
\rho_{g}(y)=\left(\begin{array}{c}
a_{0} \cdot q_{0}^{-1} * y_{A} * q_{0} \\
b_{0} \cdot q_{0}^{-1} * y_{b} * q_{0}
\end{array}\right)=\left(\begin{array}{c}
a_{0} \cdot q_{0}^{-1} *\left(a_{s} \cdot q^{-1} * A * q\right) * q_{0} \\
b_{0} \cdot q_{0}^{-1} *\left(b_{s} \cdot q^{-1} * B * q\right) * q_{0}
\end{array}\right)= & \left(\begin{array}{c}
a_{s} \cdot a_{0} \cdot q_{0}^{-1} * q^{-1} * A * \overbrace{\left(q * q_{0}\right)}^{Q} \\
b_{s} \cdot b_{0} \cdot \underbrace{\left(q_{0}^{-1} * q^{-1}\right)}_{=\left(q * q_{0}\right)^{-1} \text { i.e., } Q^{-1}} * B * \overbrace{\left(q * q_{0}\right)}^{Q}
\end{array}\right) \\
& =\left(\begin{array}{l}
a_{s} \cdot a_{0} \cdot Q^{-1} * A * Q \\
b_{s} \cdot b_{0} \cdot Q^{-1} * B * Q
\end{array}\right) \\
& =h\left(\varphi_{g}(x), \psi_{g}(u)\right)
\end{aligned}
$$

Then, if we consider the particular case where : $g=x^{-1}=\left[\left(q^{-1}\right)^{T}\left(-q * \omega_{b} * q^{-1}\right)^{T} a_{s}^{-1} b_{s}^{-1}\right]^{T}$, we obtain:

$$
\varphi_{x^{-1}}(x)=\left(\begin{array}{c}
q * q^{-1} \\
q * \omega_{b} * q^{-1}-q * \omega_{b} * q^{-1} \\
a_{s} \cdot a_{s}^{-1} \\
b_{s} \cdot b_{s}^{-1}
\end{array}\right)=\left(\begin{array}{l}
\mathbf{1} \\
0 \\
1 \\
1
\end{array}\right)=e
$$

which is equal to the neutral element $e$ of the local transformation $\varphi_{g}$. Indeed, each component of the vector $\left(\begin{array}{llll}1 & 0 & 1 & 1\end{array}\right)^{T}$ corresponds to the neutral element of:

- the quaternion Hamilton product $(\mathbf{1} *)$;

- the vector translation operation $(0+)$;

- and the real scalar product (1 •).

This vector forms an invariant and defines $x^{-1} \in G$ as the invariant Lie-group parametrization to choose. This result is known as the normalization equations solution and allows to build an invariant output error denoted by $E$. In the followings, we will use for commodity reasons the notation:

$$
\gamma(x)=\left(\begin{array}{c}
q^{-1} \\
-q * \omega_{b} * q^{-1} \\
a_{s}^{-1} \\
b_{s}^{-1}
\end{array}\right)
$$


The general form of a symmetry-preserving invariant observer can be defined such as :

$$
\dot{\hat{x}}=\overbrace{f(\hat{x}, u)}^{\text {prediction G-invariant }}+\overbrace{\sum_{i=1}^{n} \mathcal{K}_{i}\left(\psi_{\hat{x}^{-1}}, E:=\left[h\left(e=\varphi_{\hat{x}^{-1}}\left(\hat{x}^{-1}\right), \psi_{\hat{x}^{-1}}(u)\right)-\rho_{\hat{x}^{-1}}(y)\right]\right) w_{i}(\hat{x})}^{\text {invariant correction term }}
$$

In Eq.(5), $\hat{x}$ corresponds to the estimated state vector and is an element of $\mathbb{R}^{n}$. We recognize in Eq.(5) the typical mathematical expression of a nonlinear state estimator in which a model-based prediction whose dynamics is $G$-invariant and its output $G$-equivariant is summed with an invariant correction term. The idea is to build an invariant additive correction term base on the nonlinear gains $\mathcal{K}_{i}$ (which depend on the invariances $\left(e, \psi_{\hat{x}^{-1}}, \rho_{\hat{x}^{-1}}\right)$ in a nonlinear way) which keeps the whole estimated state vector dynamics $\dot{\hat{x}}$ $G$-invariant such as for the dynamical system representation $f$. In Eq.(5) the standard analytical expression of the invariant output error $E$ is given. Its explict mathematical expression can be calculated directly such that:

$$
\begin{aligned}
E & =h\left(e=\varphi_{\hat{x}^{-1}}(x), \psi_{\hat{x}^{-1}}(u)\right)-\rho_{\hat{x}^{-1}}(y) \\
& =\left(\begin{array}{l}
A-\hat{a}_{s}^{-1} \hat{q} * y_{A} * \hat{q}^{-1} \\
B-\hat{b}_{s}^{-1} \hat{q} * y_{B} * \hat{q}^{-1}
\end{array}\right)=\left(\begin{array}{c}
E_{A} \\
E_{B}
\end{array}\right)
\end{aligned}
$$

Moreover, the $w_{i}(\hat{x})$ vectors form an invariant frame i.e., for each $\hat{x} \in \mathcal{X},\left(w_{1}(\hat{x}), \cdots, w_{n}(\hat{x})\right)$ project the invariant correction term on each component of $f(\hat{x}, u)$ (i.e., the tangent state space). In our case, considering the canonical basis of $\mathbb{R}^{3},\left(e_{i}\right)_{i \in[1 ; 3]}$, the expression of these vectors which define an invariant reference frame (also called invariant natural basis in the tangent state space) is obtained by the 8 vector equations with respect to variable $w\left(\hat{q}, \hat{\omega}_{b}, \hat{a}_{s}, \hat{b}_{s}\right)$ s.t.:

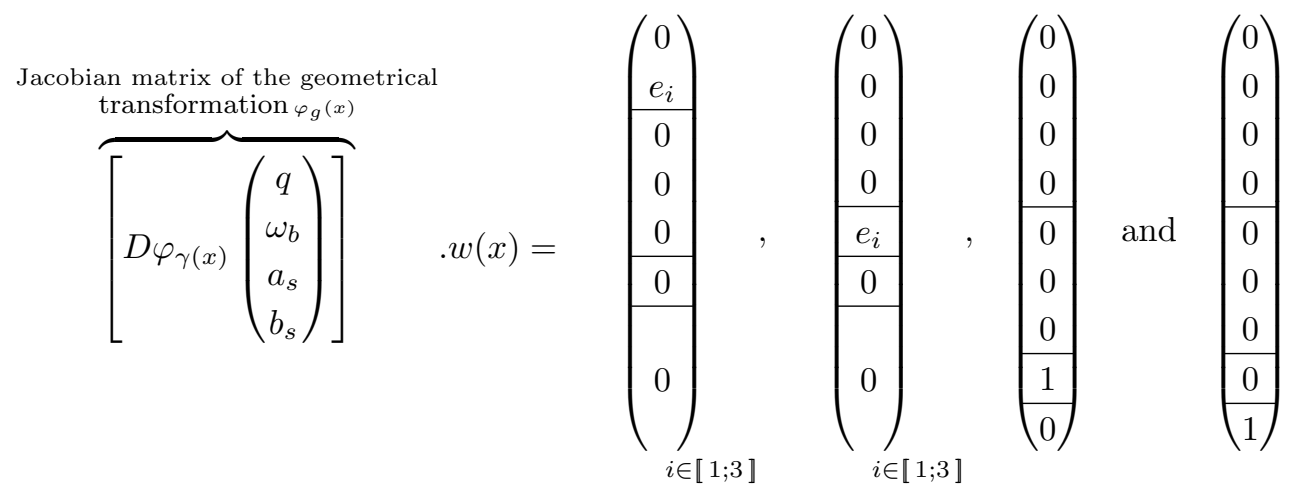

By inverting the Jacobian matrix $D \varphi_{\gamma(x)}\left(\begin{array}{c}q \\ \omega_{b} \\ a_{s} \\ b_{s}\end{array}\right)$, it follows that the invariant vector field $w_{i}(x)$ is given by:

$$
\left(\begin{array}{c}
w_{i}^{q}(x) \\
w_{i}^{\omega_{b}}(x) \\
w^{a_{s}}(x) \\
w^{b_{s}}(x)
\end{array}\right)=\left(\begin{array}{c}
0 \\
e_{i} * q \\
0 \\
0 \\
0 \\
0 \\
0
\end{array}\right)_{i \in[1 ; 3]},\left(\begin{array}{c}
0 \\
0 \\
0 \\
0 \\
\hline q^{-1} * e_{i} * q \\
0 \\
0 \\
0 \\
i \in[1 ; 3] \\
0 \\
0 \\
0 \\
0 \\
0 \\
0 \\
0 \\
0 \\
0 \\
0 \\
b_{s}
\end{array}\right) \text { and }\left(\begin{array}{c}
0 \\
0 \\
0 \\
0 \\
0 \\
0 \\
0 \\
0 \\
0 \\
0
\end{array}\right.
$$


Then, we have:

$$
\forall i \in \llbracket 1 ; n \rrbracket, \mathcal{K}_{i}\left(\psi_{\hat{x}^{-1}}(u), E=0\right)=0 \text { (i.e., no correction if no output error !) }
$$

It follows that the $1^{\text {st }}$ order Taylor series of each nonlinear gain is reduced to:

$$
\forall i \in \llbracket 1 ; n \rrbracket, \mathcal{K}_{i}\left(\psi_{\hat{x}^{-1}}(u), E\right)=\overline{\mathcal{K}}_{i}\left(\psi_{\hat{x}^{-1}}(u), E\right) \cdot E
$$

So that a linear approximation of Eq.(5) can be obtained easily s.t.:

$$
\dot{\hat{x}}=f(\hat{x}, u)+\sum_{i=1}^{n} \overline{\mathcal{K}}_{i}\left(\psi_{\hat{x}^{-1}}(u), E\right) \cdot E \cdot w_{i}(\hat{x})
$$

Combining all these results, Eq.(8) can be detailed as follows:

$$
\mathcal{O} \mathcal{M}_{s}\left\{\begin{array}{l}
\dot{\hat{q}}=\frac{1}{2} \hat{q} *\left(\omega_{m}-\hat{\omega}_{b}\right)+\sum_{i=1}^{3}\left(\overline{\mathcal{K}}_{A i}^{q} \cdot E_{A}+\overline{\mathcal{K}}_{B i}^{q} \cdot E_{B}\right) e_{i} * \hat{q} \\
\dot{\hat{\omega}}_{b}=\sum_{i=1}^{3} \hat{q}^{-1} *\left(\overline{\mathcal{K}}_{A i}^{\omega_{b}} \cdot E_{A}+\overline{\mathcal{K}}_{B i}^{\omega_{b}} \cdot E_{B}\right) * \hat{q} \\
\dot{\hat{a}}_{s}=\hat{a}_{s}\left(\overline{\mathcal{K}}_{A i}^{a_{s}} \cdot E_{A}+\overline{\mathcal{K}}_{B i}^{a_{s}} \cdot E_{B}\right) \\
\dot{\hat{b}}_{s}=\hat{b}_{s}\left(\overline{\mathcal{K}}_{A i}^{b_{s}} \cdot E_{A}+\overline{\mathcal{K}}_{B i}^{b_{s}} \cdot E_{B}\right)
\end{array}\right.
$$

where the quantities $\overline{\mathcal{K}}_{A i}^{q}, \overline{\mathcal{K}}_{B i}^{q}, \overline{\mathcal{K}}_{A i}^{\omega_{b}}, \overline{\mathcal{K}}_{B i}^{\omega_{b}}, \overline{\mathcal{K}}_{A i}^{a_{s}}, \overline{\mathcal{K}}_{B i}^{a_{s}}, \overline{\mathcal{K}}_{A i}^{b_{s}}$ and $\overline{\mathcal{K}}_{B i}^{b_{s}}$ are arbitrary $1 \times 3$ gain matrices whose choice will determine the convergence properties of the estimation scheme. A direct and analytical design can be done thanks to the linearization of the invariant state error denoted $\eta(x, \hat{x})$ and defined such as:

$$
\eta(x, \hat{x})=\varphi_{\gamma(x)}(\hat{x})-\varphi_{\gamma(x)}(x)=\left(\begin{array}{c}
\hat{q} * q-\mathbf{1} \\
\hat{q} *\left(\hat{w}_{b}-w_{b}\right) * \hat{q}^{-1} \\
a_{s} / \hat{a}_{s} \\
b_{s} / \hat{b}_{s}
\end{array}\right) \sim x^{-1} \hat{x} .
$$

Such an approach can be very tedious and non-systematic for more complex dynamical systems than the ones represented by a fully kinematic model. That's why recently, a more general EKF based method dedicated to gains calculation (exploiting a re-linearization of the invariant state estimation error dynamics) has been developed in. ${ }^{7-9}$ In that way, the Invariant EKF can be viewed as a "second-order" approximation of the invariant estimation error. Once again, this technique can be very complex to implement due to this re-linearization. As a consequence a novel and original UKF-based approach, named $\pi$-IUKF, has been defined. It addresses the approximation issue of the invariant EKF without requiring any linearization of the dynamical systems equations. It is called "pi-"IUKF because a compatibility condition, pronounced "pi", on the measurement equations has been defined and integrated to the standard UKF algorithm. This new way for calculating the matricial gains has motived our research works.

\section{B. $\pi$-IUKF algorithm}

In this section, we propose a modification of the $\mathrm{UKF}^{3}$ algorithm for nonlinear systems possessing symmetries. As mentioned in the previous section, this algorithm presents the advantage to compute an approximated solution of a nonlinear optimal filtering problem in discrete-time without requiring any linearization of the nonlinear systems equations. A method, named Sigma-Points Approach ${ }^{14}$ (SPA), is used for calculating the gaussian approximation of an unknown random variable $x_{k \mid k}$ at time $k T$ on which is applied a nonlinear transformation such as follows:

$$
\left\{\begin{array}{l}
x_{k \mid k} \sim N\left(\hat{x}_{k \mid k}, P_{k \mid k}\right) \quad \text { s.t } \quad x_{k+1}=f\left(x_{k}, u_{k}, k\right)+w_{k} \\
y_{k \mid k}=h\left(x_{k \mid k}, k\right)+v_{k}
\end{array}\right.
$$


where $x \in \mathbb{R}^{n}, y \in \mathbb{R}^{p}$ and $w, v$ are independent white gaussian noises. The aims of the basic SPA is to form a fixed number of deterministically chosen sigma-points $\chi_{k \mid k}$, which capture the "true" mean $\hat{x}_{k \mid k}$ and covariance $P_{k \mid k}$ of the original distribution $x_{k \mid k}$. This set of points must represent accurately the first and second order moments. These sigma-points are then propagated through the nonlinear functions Eq.(9) providing a cloud of evolving points. The mean $\hat{x}_{k \mid k+1}$ and estimated covariance matrix $P_{k \mid k+1}$ of the transformed points are then computed based on their statistics. Therefore, the UKF is only a straightforward extension of the SPA for computing approximate solutions to the estimation problem posed whose mathematical form is presented by:

$$
\begin{aligned}
x_{k+1} & =f\left(x_{k}, u_{k}\right)+w_{k} \\
z_{k} & =h\left(x_{k}, u_{k}\right)+v_{k}
\end{aligned}
$$

Concerning both evolution and observation noises ( $w$ and $v$ ), we assume that they enter into the system in an "invariant way" such as in. ${ }^{7}$ Noises can be considered as additive exogeneous invariant inputs and outputs, which do not change systems symmetries, if their insertion in the modeling equations relies on $G$ invariant/equivariant mathematical terms. That's why some specific operations (such as the right quaternion multiplications $* * q$ ) appear in the discrete differential equations at the beginning of $\S I V$. Note that in reference, ${ }^{7}$ the authors make an important assumption on the local diffeomorphism $\rho_{g}$ which is motivated by the fact that this latter application is not generally linear. This can raise problems in considering the measurement noises within the G-equivariant observation model. The exploitation of the UKF principles within the nonlinear invariant estimation framework required the definition of a particular condition on the observation equations, named compatibility condition, which corresponds to the application of a nonlinear function, denoted $\pi$, to the predicted measurement vector. The resulting operation can be interpreted as the construction of a set of new predicted measurements whose expressions can be projected in the natural base $w_{i}^{q}\left(\hat{x}_{k+1 \mid k}\right)$ (see $\left.\S I I I-A\right)$. Indeed, we have:

$$
\pi\left(h\left(\hat{x}_{k+1 \mid k}, u_{k}\right)\right)=\sum_{i=1}^{3} \rho_{\gamma_{\left(\hat{x}_{k+1 \mid k}\right)}}\left(h\left(\hat{x}_{k+1 \mid k}, u_{k}\right)\right) w_{i}^{q}\left(\hat{x}_{k+1 \mid k}\right) e_{i}
$$

where:

$$
\gamma\left(\hat{x}_{k+1 \mid k}\right)=\left(\begin{array}{c}
\hat{q}_{k+1 \mid k}^{-1} \\
-\hat{q}_{k+1 \mid k} * \hat{\omega}_{b_{k+1 \mid k}} * \hat{q}_{k+1 \mid k}^{-1} \\
\hat{a}_{s_{k+1 \mid k}}^{-1} \\
\hat{b}_{s_{k+1 \mid k}}^{-1}
\end{array}\right)
$$

Therefore, Eq.(12) can be detailed for $\mathcal{M}_{s}^{+}$as it follows :

$$
\pi\left(h\left(\hat{x}_{k+1 \mid k}, u_{k}\right)\right)=\left(\begin{array}{l}
\hat{a}_{s_{k+1 \mid k}} \cdot \sum_{i=1}^{3}\left(A * \hat{q}_{k+1 \mid k}\right) e_{i} \\
\hat{b}_{s_{k+1}} \cdot \sum_{i=1}^{3}\left(B * \hat{q}_{k+1 \mid k}\right) e_{i}
\end{array}\right)
$$

This compatibility condition ensures that the measurement prediction step will be led in an "invariant way" so that the Square-Root $\mathrm{UKF}^{14,15}$ (SR-UKF) gains now depend on the invariances. As a result, the $\pi$-IUKF relies on the same ideas as the usual UKF but instead of using a linear correction term based on a linear output error, it uses a geometrically adapted correction term based on an invariant output error. Likewise, the covariance matrix is not updated from an initial linear state error $\left(x_{0}-\hat{x}_{0}\right)$, but from an intrinsic initial invariant state error $\eta\left(\hat{x}_{0}, x_{0}\right)$. The stochastic differential equations corresponding to the $\pi$-IUKF for nonlinear systems possessing symmetries are given by the modifications of the initialization, observation and correction steps of the standard SR-UKF. Note that we use the square-root form of the UKF method because it avoids to perform a direct matrix square-root operation at each time $k T$. The pseudo-code of the $\pi$-IUKF algorithm is detailed in Appendix II. For commodity reasons, the addition of a given vector $u$ to each column of a matrix $M$ is noted $M \pm u$. This technique provides an increasing computational efficiency as well as a numerical stability. The SR-UKF calculations are based on three linear algebra computation techniques: - the QR decomposition (denoted: $\operatorname{qr}\{\cdot\}$ ); - the Cholesky factor updating technique (cholupdate ${ }^{16}$ within MATLAB $^{\mathrm{TM}}$ ); - and the least squares ${ }^{17}$ (MATLAB's ' ' operator). 


\section{Simulation results}

We now illustrate the performances reached by the developed $\pi$-IUKF method on the basis of simulated data provided by the ONERA ${ }^{\mathrm{d}}$ lab. As it was aforementioned, the driving perturbation noises are introduced within the G-invariant system of Eq.(10) such as follows:

$$
\left\{\begin{array}{l}
q_{k+1}=\frac{1}{2} q_{k} *\left(\omega_{m_{k}}-\omega_{b_{k}}\right)+W_{q_{k}} w_{q_{k}} * q_{k} \\
w_{b_{k+1}}=q_{k}^{-1} * W_{w_{k}} w_{w_{k}} * q_{k} \\
a_{s_{k+1}}=a_{s_{k}} W_{a_{k}} w_{a_{k}} \\
b_{s_{k+1}}=b_{s_{k}} W_{b_{k}} w_{b_{k}}
\end{array}\right.
$$

and the output noises enter the observation Eq.(11) such as:

$$
\left(\begin{array}{l}
z_{A_{k+1}} \\
z_{B_{k+1}}
\end{array}\right)=\left(\begin{array}{l}
a_{s_{k+1}} q_{k+1}^{-1} *\left(A+V_{A_{k+1}} v_{A_{k+1}}\right) * q_{k+1} \\
b_{s_{k+1}} q_{k+1}^{-1} *\left(B+V_{B_{k+1}} v_{B_{k+1}}\right) * q_{k+1}
\end{array}\right)
$$

In the last expressions, $W_{q_{k}}, W_{w_{k}}, W_{a_{k}}$ and $W_{b_{k}}$ (resp. $V_{A_{k}}$ and $V_{B_{k}}$ ) refer to the process (resp. measurement) noise covariance matrices which are similar to weighting terms. Followingly, we define two block diagonal matrices, denoted $W_{k}$ and $V_{k}$, by concatenating these covariance matrices. The experimental data are sampled with a frequency equal to $50 \mathrm{~Hz}$ which characterized the inertial measurement unit and the magnetometers. The measurements are corrupted by gaussian white noises whose standard deviations are set to: $\sigma_{\text {gyro }}=0.2 \%, \sigma_{\text {accelero }}=0.2 \mathrm{~g}$ and $\sigma_{\text {magneto }}=500 \mathrm{nT}$. In order to validate our $\pi$-IUKF filter, we have also introduced a biases vector on $\omega_{m}$ s.t. $\omega_{b}=[0.1 \mathrm{rad} / \mathrm{s} 0.05 \mathrm{rad} / \mathrm{s} 0.02 \mathrm{rad} / \mathrm{s}]^{T}$. The two positive scalar factor values are set to $a_{s}=1.2$ and $b_{s}=0.9$ respectively.

\section{A. Acceleration disturbance $(\dot{V} \neq 0)$}

First of all, we present the acceleration $\dot{V}$ in Fig. 3(a) for which the assumption made previously $(\dot{V}=0)$ may be wrong. Indeed, we can observe that this acceleration goes down and up strongly at time $20 s<t<35 \mathrm{~s}$. Thus, in Fig. 3(b), biases, as well as scaling factors, are a little bit influenced by this inconsistency since these ones are estimated while system is evolving. However, it appears that both biases and scaling factors converge quickly to their respective true constant values.

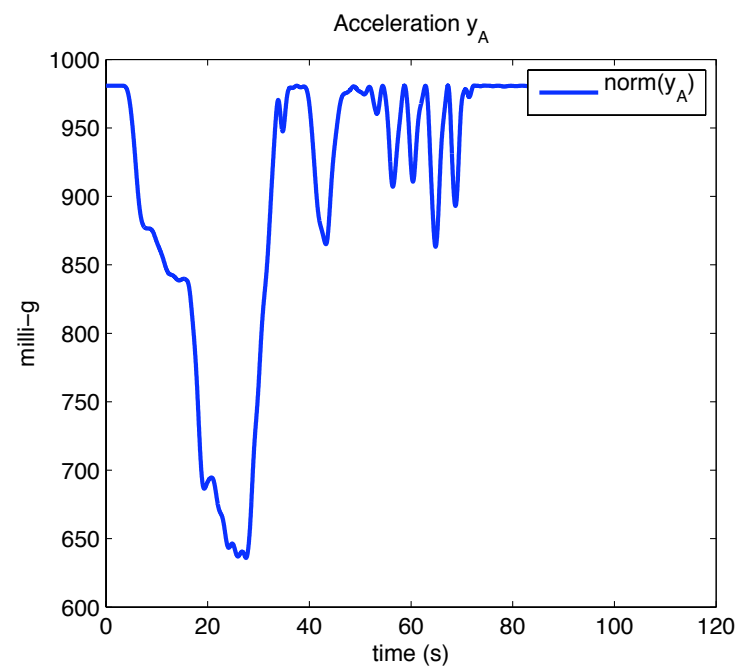

(a) Acceleration $y_{A}$ (locally: $\dot{V} \neq 0$ )

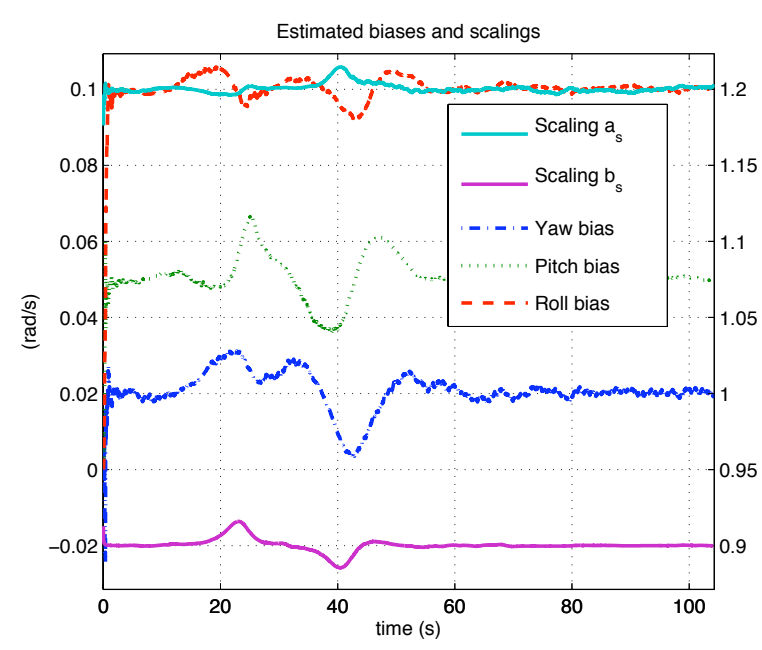

(b) Estimated biases and scaling factors

Figure 3. Biases and scaling factors locally influenced by $\dot{V} \neq 0$

\footnotetext{
${ }^{\mathrm{d}}$ Office Nationale d'Etudes et de Recherches Aérospatiales.
} 


\section{B. Attitude estimation}

In Fig. 4(a), we firstly illustrate a typical realization of the gaussian white noise stochastic processes with zero mean which perturbate the nonlinear state estimation problem to solve. Then, the real (red solid line) and estimated (blue dashed line) attitude angles $(\phi, \theta, \psi)$ are plotted on the same subfigures respectively in order to be compared. These first estimation results have been obtained without introducing an initial condition error on the state vector. We can observe on the last graph that the estimation errors are small in spite of strong acceleration phases for which the assumption $\dot{V}=0$ is not true. Moreover, we would like to see the behavior (the local domain of attraction, even if we have no proof of convergence) with an initial condition error on the estimated state vector. In Fig. 4(b), initial condition errors are now introduced on the whole state vector. For instance, the quaternion is initialized s.t. $q=\left[\begin{array}{llllll}0.8 & 0.05 & 0.05 & 0.01\end{array}\right]^{T}$ instead of $q=\left[\begin{array}{llll}1 & 0 & 0 & 0\end{array}\right]^{T}$ which is not an unit norm quaternion. The real (red solid line) and estimated (blue dashed line) Euler angles are represented and a zoom on the first seconds is depicted. We can observe the good convergence behavior of our developed $\pi$-IUKF algorithm, in terms of accuracy and settling time, in presence of process and measurement noises, sensors imperfections and erroneous initial conditions.
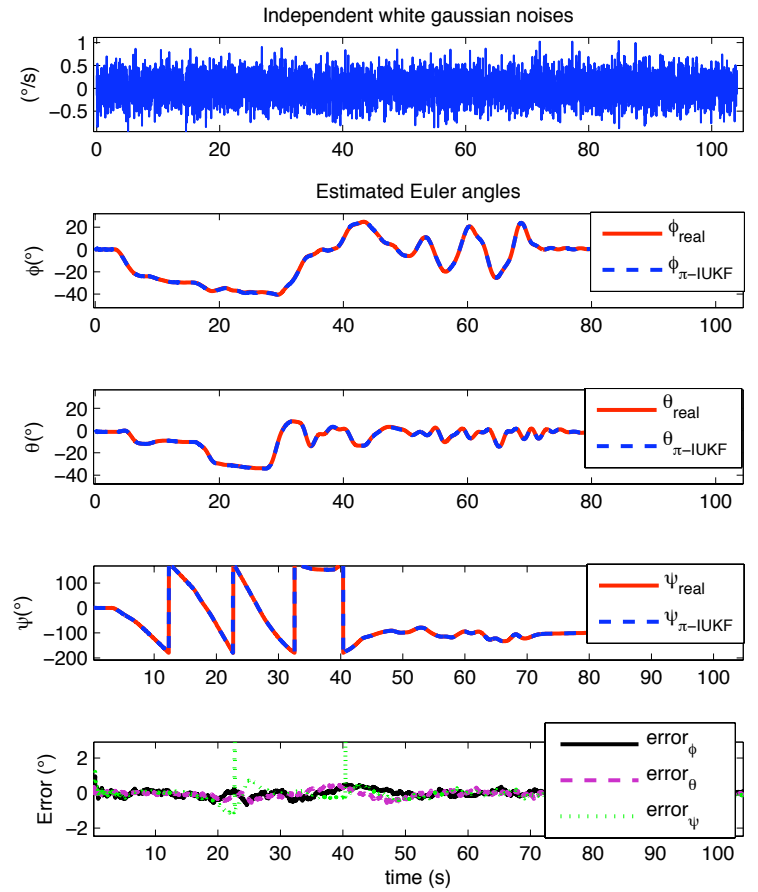

(a) Results obtained with no state initial error
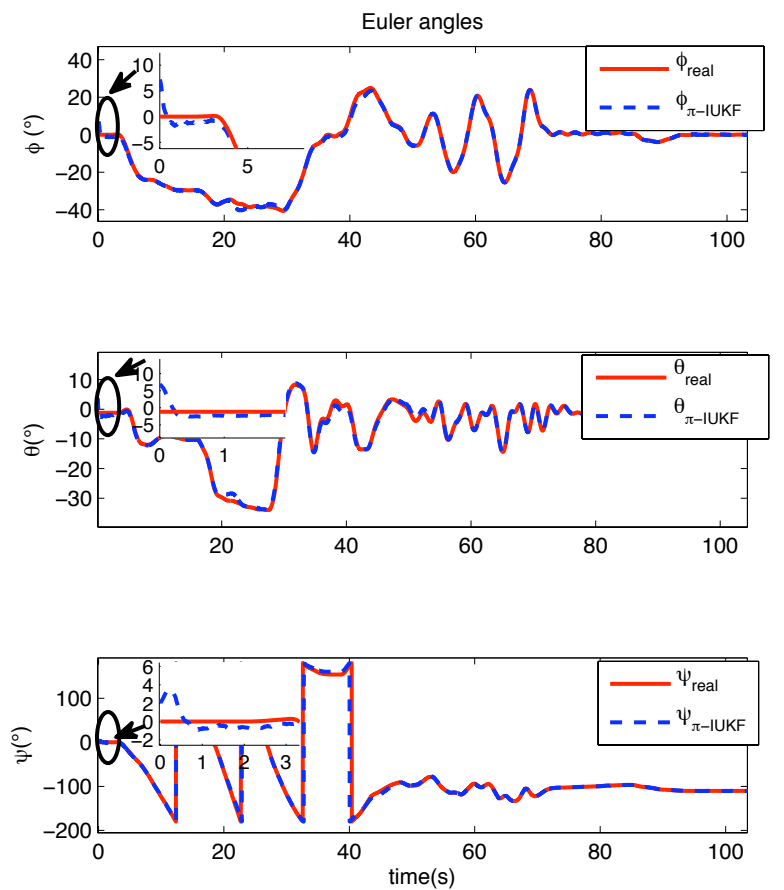

(b) Results obtained with state initial error

Figure 4. Attitude estimation results - $(\phi, \theta, \psi)$

\section{Estimated covariance matrices $P(t)$ of the $\pi$-IUKF vs. standard UKF}

Fig. 5 shows the estimation error covariance matrix $P(t)$ calculated by the standard SR-UKF method (on the left) and by the proposed $\pi$-IUKF technique. As explained in the theoretical development part, the new $\pi$-IUKF invariant observer must have the same invariance properties as, for instance, the IEKF developed in. ${ }^{7}$ If the $\pi$-IUKF estimation error covariance matrix is constant then the whole approach will be validated. The results depicted in Fig. 5 show that $P(t)$ (which is initialized with a given value $P(0)$ ) converge, as expected, to constant values over the whole flight trajectory due to the symmetry-preserving property provided by the nonlinear estimation theory based on invariant filter. Meanwhile, the SR-UKF estimation error covariance matrices do not convergence to constant values. Obviously, they also depend on the followed trajectory which is illustrated by the peaks. This is indicative of a smaller convergence domain 
for this estimation technique in comparison with the new $\pi$-IUKF method. A last cross-comparison of the results show that in some steady-state interval, the values of the standard SR-UKF and $\pi$-IUKF covariance matrices are equal. This invariance property is remarkable and can be potentially exploited to characterize some flight parameters in terms of estimation acuracy which is constant for all trajectories. Indeed, these constant covariances can be viewed as a very useful characterization of the attitude angles in a complete and future robust control law design.
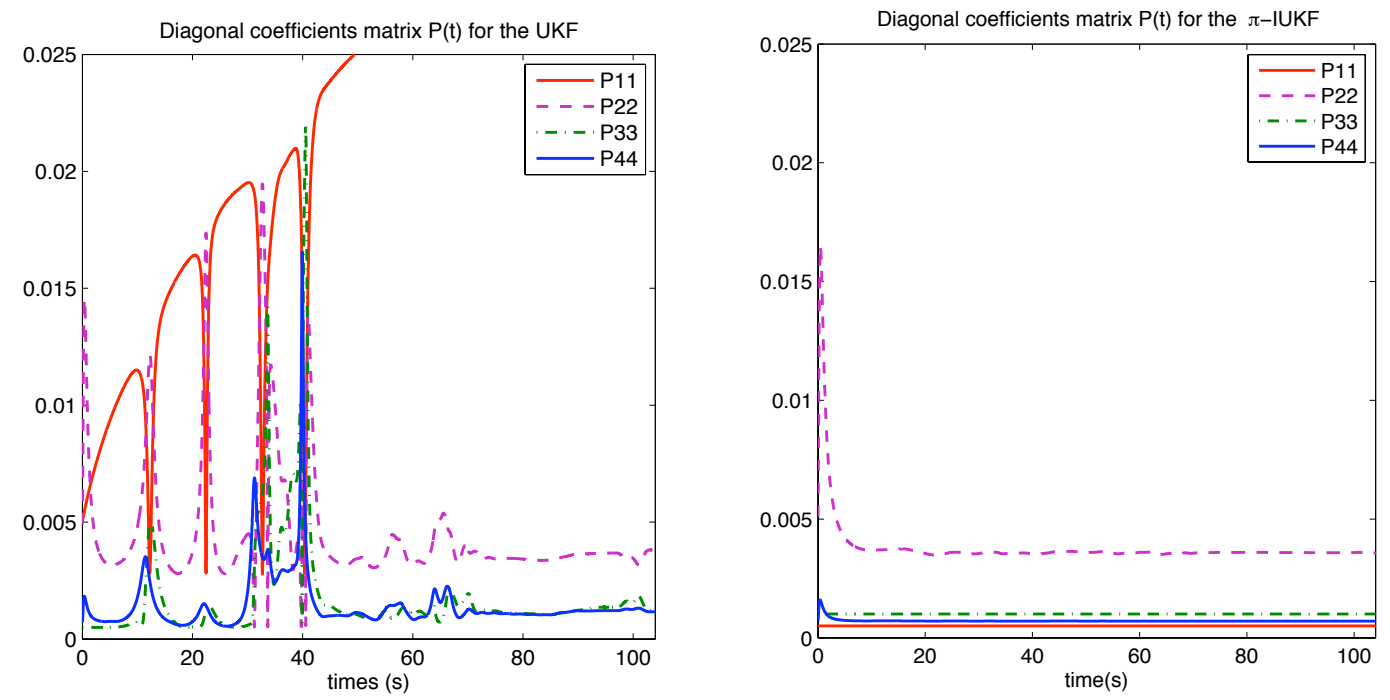

Figure 5. Estimated covariance matrices $P(t)$ : SR-UKF (left) vs. $\pi$-IUKF (right)

\section{Conclusions and Perspectives}

The new nonlinear state estimation approach named $\pi$-IUKF has been presented in this paper. The first results obtained in simulation appear as promising. The hybridation of the standard SR-UKF with the invariant observer theory represents a significant research topic to improve the handling qualities of mini-UAVs. Indeed, the computational cost of such an approach seems suitable given the low-cost sensor technology used so far and whose micro-processors performances increase year after year. The perspectives of these research works are numerous and various. In the future, a complete validation of this method must be led by treating the case of real data which will be gathered from real flight experimentations. Moreover, as the final objective concerns the insertion of mini-UAVs in complex environments (such as urban areas or indoor flights), future works will deal with the robustness of this developed methodology when one or several measurement signals become unavailable. In that case, some adaptations of the $\pi$-IUKF algorithm must be made in order to recover satisfying performances. These latter works aim at providing to the estimation scheme adaptive features.

\section{Acknowledgments}

The autors of this paper thank Thierry Le Moing from the ONERA, Toulouse, France and Prof. Erwan Salaun from the Georgia Institute of Technology, USA, for their valuable helps about nonlinear estimation theory. This research is financed by the european project "D3CoS" (see: http://www.d3cos.eu/). 


\section{Appendix I: quaternions and rotations}

An unit quaternion provide a convenient mathematical notation and a global parametrization for representing orientation and rotation of a rigid body in three dimensions. Indeed, for any unit quaternion

$$
q=q_{0}+\mathbf{q}=\cos \frac{\theta}{2}+\mathbf{u} \sin \frac{\theta}{2}
$$

and for any vector $\mathbf{p} \in \mathbb{R}^{3}$ the action of the operator

$$
q^{-1} * \mathbf{p} * q=R_{q} \cdot \mathbf{p}
$$

is associated to a rotation matrix $R_{q} \in S 0(3)$ which is a rotation of the coordinate frame about axis $\mathbf{u}=\frac{\mathbf{q}}{\|\mathbf{q}\|}$ through an angle $\theta$.

But how can a quaternion, which lives in $\mathbb{R}^{4}$, operate on a vector, which lives in $\mathbb{R}^{3}$ ?

Note that a vector $\mathbf{p} \in \mathbb{R}^{3}$ can be viewed as a pure quaternion whose real part is zero $\left(\begin{array}{l}0 \\ \mathbf{p}\end{array}\right)$. Thus, when the real part is a scalar denoted $p_{0} \in \mathbb{R}$ the quaternion $p$ is given as :

$$
p=\left(\begin{array}{c}
p_{0} \\
\mathbf{p}
\end{array}\right)
$$

We can utilize the inner product and cross product of two vector in $\mathbb{R}^{3}$ to write the above (non commutative) quaternion product $*$ in more concise form:

$$
p * q \doteq\left(\begin{array}{c}
p_{0} q_{0}-\mathbf{p} \cdot \mathbf{q} \\
p_{0} \mathbf{q}+q_{0} \mathbf{p}+\mathbf{p} \times \mathbf{q}
\end{array}\right)
$$

The unit element is $e \doteq\left(\begin{array}{l}1 \\ 0 \\ 0 \\ 0\end{array}\right)$, and $(p * q)^{-1}=q^{-1} * p^{-1}$

We have to useful formulas

$$
\begin{array}{r}
p \times q \doteq \mathbf{p} \times \mathbf{q}=\frac{1}{2}(p * q-q * p) \\
(\mathbf{p} \cdot \mathbf{q}) \mathbf{r}=-\frac{1}{2}(p * q-q * p) * r
\end{array}
$$




\section{Appendix II: $\pi$-IUKF algorithm}

\section{The pseudo-code for the $\pi$-UKF with additive noise:}

- parameters definition:

$\star$ the scalar $\lambda$ : is a parameter set to capture the moments of the given distribution.

$\star \sqrt{P}_{k \mid k}$ : is shorthand notation for a matrix $S_{k \mid k}$ such that $P_{k \mid k}=S_{k \mid k} S_{k \mid k}^{T}$.

$\star \mathcal{W}_{(m)}, \mathcal{W}_{(c)}:$ are weights associated to the mean and covariance distributions s.t : $\sum_{i=0}^{2 n} \mathcal{W}_{(m, c)}^{(i)}=1$.

$\star W_{k}, V_{k}:$ are the predifined covariance matrices of the process and observation noises.

- Initialization:

$$
\hat{x}_{0}=\mathbb{E}\left[x_{0}\right] \quad S_{0}=\operatorname{chol}\left\{\mathbb{E}\left[\eta\left(\hat{x}_{0}, x_{0}\right) \eta\left(\hat{x}_{0}, x_{0}\right)^{T}\right]\right\}
$$

- For $k T=1, \ldots, \infty$ :

1. Calculate sigma-points:

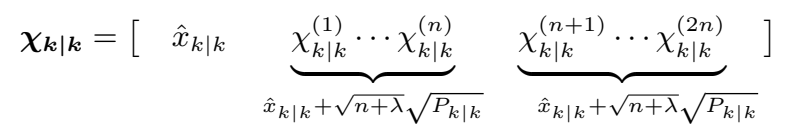

2. Time-update equations:

$$
\begin{aligned}
& \chi_{k+1 \mid k}=\left[\chi_{k+1 \mid k}^{(0)} \cdots \chi_{k+1 \mid k}^{(2 n+1)}\right]=f\left(\chi_{k \mid k}, u_{k}\right) \\
& \hat{x}_{k+1 \mid k}=\sum_{i=0}^{2 n} \mathcal{W}_{(m)}^{(i)} \chi_{k+1 \mid k}^{(i)} \\
& S_{k+1 \mid k}=\operatorname{qr}\left\{\left[\sqrt{\mathcal{W}_{(c)}^{(1)}}\left(\left[\chi_{k+1 \mid k}^{(1)} \cdots \chi_{k+1 \mid k}^{(2 n)}\right]-\hat{x}_{k+1 \mid k}\right) \sqrt{W_{k}}\right]\right\} \\
& S_{k+1 \mid k}=\text { cholupdate }\left\{S_{k+1 \mid k}, \chi_{k+1 \mid k}^{(0)}-\hat{x}_{k+1 \mid k}, \mathcal{W}_{(c)}^{(0)}\right\}
\end{aligned}
$$

3. Measurement-update equations:

$$
\begin{aligned}
& \hat{Z}_{\boldsymbol{k}+\mathbf{1} \mid \boldsymbol{k}}=\left[z_{k+1 \mid k}^{(0)} \cdots z_{k+1 \mid k}^{(2 n+1)}\right]=\pi\left(h\left(\chi_{\boldsymbol{k}+\mathbf{1} \mid \boldsymbol{k}}, u_{k}\right)\right) \\
& \hat{z}_{k+1 \mid k}=\sum_{i=0}^{2 n} \mathcal{W}_{(m)}^{(i)} z_{k+1 \mid k}^{(i)} \\
& S_{z, k+1 \mid k}=q r\left\{\left[\sqrt{\mathcal{W}_{(c)}^{(1)}}\left[\hat{\boldsymbol{Z}}_{\boldsymbol{k}+\mathbf{1} \mid \boldsymbol{k}}(:, 2: 2 n+1)-\hat{z}_{k+1 \mid k}\right] \sqrt{V_{k+1}}\right]\right\} \\
& S_{z, k+1 \mid k}=\operatorname{cholupdate}\left\{S_{z, k+1 \mid k}, z_{k+1 \mid k}^{(0)}-\hat{z}_{k+1 \mid k}, \mathcal{W}_{(c)}^{(0)}\right\} \\
& P_{x z, k+1 \mid k}=\sum_{i=0}^{2 n} \mathcal{W}_{(c)}^{(i)}\left[\chi_{k+1 \mid k}^{(i)}-\hat{x}_{k+1 \mid k}\right]\left[z_{k+1 \mid k}^{(i)}-\hat{z}_{k+1 \mid k}\right]^{T}
\end{aligned}
$$

4. Compute the Kalman filter gain:

$$
\begin{aligned}
& \overline{\mathcal{K}}_{k+1}=\left(P_{x z, k+1 \mid k} / S_{z, k+1 \mid k}^{T}\right) / S_{z, k+1 \mid k} \\
& \hat{x}_{k+1 \mid k+1}=\hat{x}_{k+1 \mid k}+\overline{\mathcal{K}}_{k+1}(\pi, E) \cdot E \cdot w_{i}^{q}\left(\hat{x}_{k+1 \mid k}\right) \\
& U=\overline{\mathcal{K}}_{k+1} S_{z, k+1 \mid k} \\
& S_{k+1 \mid k+1}=\text { cholupdate }\left\{S_{k+1 \mid k}, U,-1\right\}
\end{aligned}
$$




\section{References}

${ }^{1}$ Crassidis, J.L., Markley, F.L., Cheng, Y., "A survey of Nonlinear Attitude Estimation Methods," Journal of Guidance Control and Dynamics, vol.30, no.1, pp.12-29, 2007.

${ }^{2}$ Xiong, K., Zhang, H.Y., "Performance evaluation of UKF-based nonlinear filtering," Automatica, vol. 42, no. 2, pp.261$270,2006$.

${ }^{3}$ Julier, S. J., and Uhlmann, J. K., "The Unscented Kalman Filter," Proceeding of the IEEE, vol. 92, no. 3, pp. 401-422, 2004.

${ }^{4}$ van der Merwe, R., "Sigma-point Kalman filters for probabilistic inference in dynamic state-space models," Ph.D., Portland, OR: OGI School of Sci. Eng., Oregon Health and Sci. Univ., 2004.

${ }^{5}$ Sarkka, S., "On Unscented Kalman Filtering for State Estimation of Continous-Time Nonlinear Systems," IEEE Trans. Automat. Control, vol. 52, no. 9, pp.1631-1641, 2007.

${ }^{6} \mathrm{Xu}$, J., Wang, S., Dimirovski, G.M, Jing, Y., "Stochastic Stability of the Continuous-Time Unscented Kalman Filter," Proc. of the 47th IEEE Conf. on Decision and Control, 2008, pp. 5110-5115.

${ }^{7}$ Bonnabel, S. , Martin, P., and Salan, E., "Invariant Extended Kalman Filter:Theory and Application to a Velocity-Aided Attitude Estimation Problem," Proc. of the 48th IEEE Conf. on Decision and Control, 2009, pp.1297-1304.

${ }^{8}$ Barczyk, M., F. Lynch, A., "Invariant Extended Kalman Filter Design for a Magnetometer-plus-GPS Aided Inertial Navigation System," Proc. of the 50th IEEE Conf. on Decision and Control and European Control Conference, 2011, pp. 5389-5394.

${ }^{9}$ Martin, P., Salan, E., "Generalized Multiplicative Extended Kalman Filter for Aided Attitude and Heading Reference System," Proc. of the 2010 AIAA Guidance, Navigation, and Control Conference, 2010, AIAA 2010-8300.

${ }^{10}$ Farrell, J. and Barth, M., The global positioning system and inertial navigation, McGraw-Hill, 1998.

${ }^{11}$ Barbour, N., Schmidt, G. "Inertial Sensor Technology Trends," IEEE Sensors Journal, vol. 1, No.4, 2001, pp. 332-339.

${ }^{12}$ Salan, E., "Filtering Algoritms and Avionics Systems for Unmanned Aerial Vehicles," PhD theses, Mines ParisTech, 2009.

${ }^{13}$ Bonnabel,S., Martin, P., and Rouchon, P., "Non-Linear Symmetry-Preserving Observers on Lie Groups," IEEE Trans. Automat. Control, vol. 54, no. 7, pp. 1709-1713, 2009.

${ }^{14}$ van der Merwe, R., Wan, E., and Julier, S. J., "Sigma-point Kalman Filters for Nonlinear Estimation and Sensor-Fusion: Applications to Integrated Navigation," Proceedings of the AIAA Guidance, Navigation ES Control Conference, 2004, pp. $5120-5150$.

${ }^{15}$ van der Merwe, R., Wan, E., "The Square-Root Unscented Kalman Filter for State and Parameter-Estimation," Proc. of the IEEE International Conf. on Acoustics, Speech, and Signal Processing, 2001, pp. 3461-3464.

${ }^{16}$ Mathworks. MATLAB - the language of technical computing. http ://www.math-works.fr/products/matlab/.

${ }^{17}$ Press, W.H., Teukolsky, S.A., Vetterling, W.T., Flannery, B.P.,Numerical Recipes in C : The Art of Scientific Computing, Cambridge University Press, 2 edition, 1992. 